\title{
Comparison between Visualization of Microcalcifications by Digital Breast Tomosynthesis and Full-Field Digital Mammography
}

\author{
Ryusuke Murakami ${ }^{1}$, Hitomi Tani ${ }^{1}$, Izumi Miki ${ }^{1}$, Tamiko Yoshida ${ }^{1}$, \\ Shinichiro Kumita1, Nachiko Uchiyama² \\ ${ }^{1}$ Department of Radiology, Nippon Medical School Hospital, Tokyo, Japan \\ ${ }^{2}$ Department of Radiology, National Cancer Center, Tokyo, Japan \\ Email: rywakana@nms.ac.jp
}

How to cite this paper: Murakami, R., Tani, H., Miki, I., Yoshida, T., Kumita, S. and Uchiyama, N. (2020) Comparison between Visualization of Microcalcifications by Digital Breast Tomosynthesis and FullField Digital Mammography. Open Journal of Radiology, 10, 90-100.

https://doi.org/10.4236/ojrad.2020.102010

Received: May 19, 2020

Accepted: June 12, 2020

Published: June 15, 2020

Copyright $\odot 2020$ by author(s) and Scientific Research Publishing Inc. This work is licensed under the Creative Commons Attribution International License (CC BY 4.0).

http://creativecommons.org/licenses/by/4.0/

Open Access

\begin{abstract}
Objective: To date, few studies have compared the diagnostic performance and visibility of microcalcifications obtained using digital breast tomosynthesis (DBT) with those obtained from full-field digital mammography (FFDM). The visualization and characterization of microcalcifications with DBT remain controversial. The purpose of this study was to compare the visibility of microcalcifications and determine whether DBT exhibits a diagnostic advantage for visualizing microcalcifications over FFDM. Methods: We retrospectively reviewed 120 cases including DBT and FFDM imaging (60 histologically verified as breast cancers and 60 as benign microcalcifications or normal). DBT images with a wide scan-angle of $50^{\circ}$ and FFDM images were obtained using a flat-panel system (MAMMOMAT Inspiration, Siemens). Images were independently reviewed by four boardcertified radiologists and evaluated for the presence of microcalcifications, probability of malignancy (BI-RADS classification), and visibility. Results: In predicting the malignancy of detected microcalcifications, no significant difference was found between readers' areas under the receiver operating characteristic curve for DBT and FFDM $(\mathrm{p}=0.068)$. The visibility scores of detected microcalcifications were $3.74 \pm 1.06$ for DBT and $3.46 \pm 0.93$ for FFDM, respectively. The visibility of microcalcifications when using DBT was found to be significantly superior to that of FFDM $(p<0.05)$. Conclusion: Our results suggest that the image quality of DBT with a wide scanangle is comparable or superior to that obtained with FFDM in terms of both visibility and assessment of microcalcifications.
\end{abstract}




\section{Keywords}

Breast, Breast Cancer, Microcalcifications, Digital Mammography, Digital Breast Tomosynthesis

\section{Introduction}

Digital breast tomosynthesis (DBT) is an increasingly used technique for both breast cancer screening and assessment, and it has shown promise in improving the visualization and characterization of lesions [1] [2] [3]. DBT is a 3-dimensional (3D) X-ray breast imaging method wherein high spatial resolution tomographic images of the breast are reconstructed from multiple low-dose projection images by rotating an X-ray tube in an arc around a digital detector. DBT provides information regarding the $3 \mathrm{D}$ aspect of the underlying tissue by imaging the breast in thin slices so that tissue is not superimposed. The advantage of DBT in the evaluation of mass, asymmetry, and architectural distortions has been well known, with reproduced findings showing them exceeding the worth of conventional mammographic images [4]. DBT shows a higher detection rate and diagnostic accuracy for both benign and malignant mass-like lesions, with better sensitivity and specificity and lower recall rates [5]. The combination of FFDM and DBT improved breast cancer accuracy, decreased the false-negative rate, and increased the sensitivity relative to using only FFDM [6]-[11]. However, this does not apply to the assessment of microcalcifications and the detectability of microcalcifications using DBT remains controversial.

Mammography is considered the most important diagnostic tool in the detection and characterization of microcalcifications. Suspicious malignant microcalcifications usually present with specific morphologic features [12]. Approximately $40 \%$ of breast cancers present microcalcifications [13], and more $90 \%$ of ductal carcinoma in situ (DCIS) are diagnosed by the visualization of microcalcifications on mammography [14]. A careful characterization of microcalcifications morphology and distribution is essential to stratify the risk of malignancy and to guide clinical management decisions, such as core needle biopsy or vacuum-assisted biopsy.

There are a few studies that mention the value of DBT in the visualization and characterization of microcalcifications [15] [16] [17] [18] [19]. There is a discrepancy of opinions regarding the value of DBT. Some authors suggest that DBT underestimate microcalcification cluster classification relative to FFDM [16] [17] [18] [19]. Therefore, recent studies have shown no definitive consensus regarding the use of DBT in characterizing and detecting microcalcifications. These studies opened the discussion about whether DBT is suitable for the investigation of microcalcifications. Notably, most studies used acquisition parameters characterized by relatively narrow scan angles and repetitively evaluated DBT in association with 2D imaging [16] [17] [18]. Conversely, to date, the 
evaluation of microcalcifications using DBT with a wide scan-angle has been scarce.

The scan-angle, along with the number of projections and distribution, is one of the main acquisition parameters that affect the image quality of DBT [20] [21]. The scan-angle is characterized by a high variability depending on the device, ranging from $15^{\circ}$ (narrow angle) to $50^{\circ}$ (wide angle). A wider angular range is expected to enhance in-depth resolution. However, the optimal combination with various acquisition parameters is currently a topic of intense discussion [20].

The purpose of this study is to assess the performance of DBT with a wide scan-angle for the detection and characterization of microcalcifications and to compare the visibility of microcalcifications by DBT with that on FFDM, and to investigate whether the information of DBT has an impact on the diagnostic advantage for microcalcifications.

\section{Material and Methods}

\subsection{Case Selection}

The institutional ethics committee approved this retrospective study. The need for informed consent was waived. Inclusion criteria for the microcalcification group were as follows: 1) availability of images from at least one breast with two views in FFDM and DBT examinations; 2) histopathological verification of microcalcifications; and 3) From January 2014 to December 2016, 818 patients underwent combined FFDM and DBT for diagnostic purposes. Finally, 246 examinations performed in 244 patients (age, 26 - 82 years; mean age, 51 years) were selected. A radiologist (N.U), who was not involved in interpreting for the study, reviewed for appropriate case selection to create the data set.

The study cohort included 60 cases that were verified to have breast cancer by histopathology and 47 cases of microcalcifications assessed as benign following image-guided biopsy (Table 1). Propensity score matching was used to match the cancer group in a 1:1 ratio with 13 normal cases from the screening examinations performed at our institution during the study period. Normal cases were defined by a final BI-RADS (Breast Imaging Reporting and Data System) score of 1. Age and mammographic density were considered for matching. The final study cohort included 120 patients. This selection process did not necessarily cover the full range of lesions usually encountered in a clinical setting.

\subsection{Image Acquisition}

The patients underwent FFDM and DBT imaging of both breasts in the craniocaudal (CC) and mediolateral oblique (MLO) positions using a standard DBT system (Mammomat Inspiration, Siemens, Erlangen, Germany), that acquires 25 projections with a scan-angle of $50^{\circ}$. Two bilateral views were obtained in combo mode (acquisition of 2D plus 3D images in the same session). An anode/filter combination $\mathrm{W} / \mathrm{Rh}$ was used with the same tube voltage as that for FFDM. 
Table 1. Histology results of the 107 cases that presented with microcalcifications selected for the study.

\begin{tabular}{llc}
\hline \multicolumn{1}{c}{ Histology } & N \\
\hline Malignant & Ductal carcinoma in situ & 38 \\
& Invasive ductal carcinoma & 19 \\
& Invasive lobular carcinoma & 1 \\
& Mucinous carcinoma & 1 \\
& Invasive micropapillary carcinoma & 1 \\
& & $(60)$ \\
Benign & Fibrocystic changes & 23 \\
& Atypical ductal hyperplasia & 3 \\
& Sclerosing adenosis & 3 \\
& Papilloma & 2 \\
& Hyperplasia without atypia & 2 \\
Fibroadenoma & 2 \\
& Unspecified benign lesions & 12 \\
& & $(47)$ \\
& & 107 \\
\hline
\end{tabular}

These images were automatically reconstructed into a series of $1 \mathrm{~mm}$-thick slices using the filtered back projection technique.

\subsection{Image Assessment}

Four radiologists specialized in breast imaging performed the readings (R.M., H.T., T.Y., and I.M.). All readers had at least 8 years of clinical experience with DBT. Readers were aware of the aim of the study, but they were blinded to the presence and type of lesions, patients' clinical history, or diagnosis.

All images were analyzed using a dedicated digital mammography workstation (Plissimo MG, Panasonic, Osaka, Japan) with a set of 5-MP monochrome LCD monitors (MFGD5621HD, $2048 \times 2560$ pixels, 21.3 inch; BARCO, Torhout, Belgium). Each reader conducted a separate analysis in a routine mammography reading room. Every reader performed two reading sessions. During each reading session, all the patients were randomized to 60 cases with FFDM and 60 cases with DBT. DBT readings were performed by scrolling through the examination. In addition, readers were allowed to use the magnification function for both FFDM and DBT. Additional images (e.g., magnification views) were not included in the present study. Similarly, slabs or other reconstructed images were not available. To minimize the effects of learning and memory, a time gap of least 4 weeks was imposed between reading sessions.

During interpretation, readers were asked to indicate whether they detected microcalcification clusters. Readers were instructed to grade according to the 
American College of Radiology Breast Imaging reporting and data system (BI-RADS) lexicon. Subsequently, readers were asked to assign rating on visibility (from $0=$ no microcalcifications to $5=$ microcalcifications clearly visible). Visibility of microcalcifications was defined as sharper visualization with better contrast against the background of breast parenchyma.

\subsection{Statistical Analysis}

All statistical calculations were performed on a per breast basis (taking the most suspicious finding into consideration) using Ekuseru-Toukei 2015 (Social Survey Research Information Co., Ltd., Japan). For the detection of microcalcifications and the prediction of malignancy, BI-RADS scores were evaluated with a receiver operating characteristic (ROC) analysis of variance. Curve fitting followed a nonparametric trapezoidal method. Areas under the ROC curve (AUC) were computed for each reader in each mode. To evaluate microcalcification conspicuity and visibility, visibility scores of the microcalcifications detected with FFDM and DBT were compared by paired t-test for each reader. A p-value of less than 0.05 (two-sided) was considered statistically significant.

\section{Results}

\subsection{Characteristics of the Microcalcifications}

The distribution of microcalcifications among 107 cases was diffuse or regional in 10 cases (9.3\%), grouped in 52 (48.6\%), and segmental in 45 (42.1\%). Microcalcifications were classified as having coarse heterogeneous shape in 28 cases (26.2\%), amorphous shape in 57 (53.3\%), fine pleomorphic shape in $14(13.1 \%)$, and fine linear shape in $8(7.4 \%)$.

\subsection{Average Glandular Dose (AGD)}

The mean compressed breast thickness was $42.1 \mathrm{~mm}$ and $39.1 \mathrm{~mm}$ for CC and MLO views, respectively. The mean AGD values in FFDM were $1.82 \mathrm{mGy}$ and $1.94 \mathrm{mGy}$ for CC and MLO views, respectively. The mean AGD values in DBT were $2.53 \mathrm{mGy}$ and $2.63 \mathrm{mGy}$ for CC and MLO views, respectively (Table 2).

Table 2. Average glandular dose (AGD) for each projection in FFDM and DBT imaging after correction for the actual breast composition.

\begin{tabular}{cccc}
\hline Projection & CBT $(\mathrm{mm})$ & AGD of FFDM (mGy) & AGD of DBT (mGy) \\
\hline RCC & $42.3 \pm 11.7(21-86)$ & $1.03 \pm 0.26(0.66-1.88)$ & $1.54 \pm 0.49(0.93-2.61)$ \\
LCC & $42.0 \pm 11.0(17-69)$ & $1.00 \pm 0.25(0.59-1.86)$ & $1.52 \pm 0.36(0.88-2.68)$ \\
RMLO & $39.0 \pm 11.2(18-78)$ & $1.03 \pm 0.25(0.60-1.94)$ & $1.54 \pm 0.45(0.90-2.68)$ \\
LMLO & $39.1 \pm 10.8(15-69)$ & $1.02 \pm 0.26(0.58-1.86)$ & $1.52 \pm 0.38(0.87-2.80)$ \\
\hline
\end{tabular}

Data are presented as mean \pm SD (min.-max.). RCC: right craniocaudal, LCC: left craniocaudal, RMLO: right mediolateral oblique, LMLO: left mediolateral oblique, CBT: compressed breast thickness. 


\subsection{Diagnostic Performance and Descriptors}

Results of the ROC analysis based on the BI-RADS score are shown in Table 3. The mean AUC was 0.810 (range, 0.736 - 0.885) for DBT and 0.778 (range, 0.715 - 0.842) for FFDM. When comparing ROC curves, no significant differences between modalities were found between readers $(\mathrm{P}=0.148)$ (Table 3 ).

Diagnostic accuracy was $78.3 \%$ (mean; range, 71.0 - 82.2) for DBT and $70.6 \%$ (mean; range, 66.4 - 95.2) for FFDM. The sensitivity was 63.3 - 88.3 for DBT and $55.7-81.7$ for FFDM. The specificity was $84.5-88.7$ for DBT and $83.0-89.4$ for FFDM.

\subsection{Visibility}

The visibility scores for microcalcifications with FFDM and DBT is shown in Table 4. Visibility score was $3.74 \pm 1.06$ for DBT and $3.46 \pm 0.93$ for FFDM. The visibility of DBT was significantly superior to that for FFDM $(\mathrm{P}=0.019)$. For malignant lesions, the DBT score was also significantly higher than the FFDM score $(\mathrm{P}<0.05)$ (Figure 1, Figure 2). Of note, DBT scores for malignant lesions were significantly higher than those for benign lesions $(4.08 \pm 0.93$ vs. $3.29 \pm$ 1.06; $\mathrm{P}<0.05)$.

\section{Discussion}

The main purpose of this study was to understand whether microcalcifications, once identified, are better seen with DBT. Our results demonstrate that microcalcifications of all types are seen as well or with greater clarity on DBT studies than on 2D mammography FFDM.

The accurate detection and evaluation of microcalcifications are important for

Table 3. Readers' AUCs based on the BI-RADS score for FFDM and DBT.

\begin{tabular}{ccccccc}
\hline & \multicolumn{3}{c}{ FFDM } & \multicolumn{3}{c}{ DBT } \\
\cline { 2 - 7 } & AUC & S.E & $95 \%$ CI & AUC & S.E & $95 \%$ CI \\
\hline Reader 1 & 80.5 & 3.8 & $73.0-87.8$ & 85.5 & 3.6 & $78.5-92.5$ \\
Reader 2 & 74.5 & 4.2 & $66.2-82.7$ & 79.4 & 3.9 & $71.8-86.9$ \\
Reader 3 & 82.1 & 3.5 & $75.3-88.8$ & 84.1 & 3.3 & $77.5-90.6$ \\
Reader 4 & 74.4 & 4.0 & $66.5-82.3$ & 75.3 & 4.1 & $67.3-83.2$ \\
\hline
\end{tabular}

FFDM: full-field digital mammography, DBT: digital breast tomosynthesis, AUC: area under the curve, CI: confidence interval, S.E.: standard error.

Table 4. Mean visibility score ratings for FFDM and DBT.

\begin{tabular}{cccc}
\hline & FFDM & DBT & \\
\hline Total microcarcifications & $3.46 \pm 0.93$ & $3.74 \pm 1.06$ & $\mathrm{P}=0.019^{*}$ \\
Malignant lesions & $3.73 \pm 0.92$ & $4.08 \pm 0.93$ & $\mathrm{P}=0.025^{*}$ \\
Benign lesions & $3.11 \pm 0.84$ & $3.29 \pm 1.06$ & $\mathrm{P}=0.335$ \\
\hline
\end{tabular}

Data are presented as mean \pm SD. 


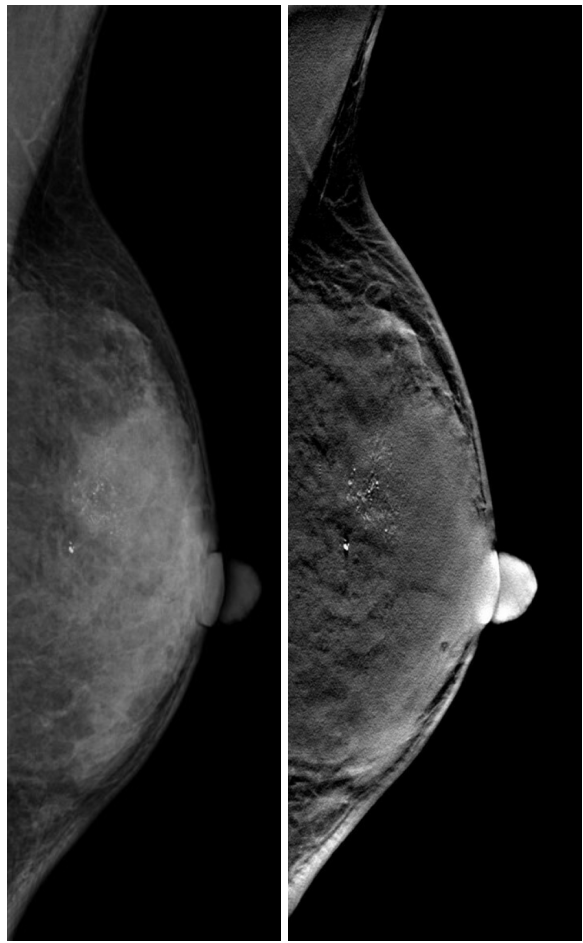

Figure 1. Images from a 49-year-old woman with ductal carcinoma in situ show amorphous to fine pleomorphic calcifications in a segmental distribution in the left breast. (Left) Full-field digital mammography (FFDM). (Right) The digital breast tomosynthesis (DBT) study shows the calcification more clearly as seen on this single slice.
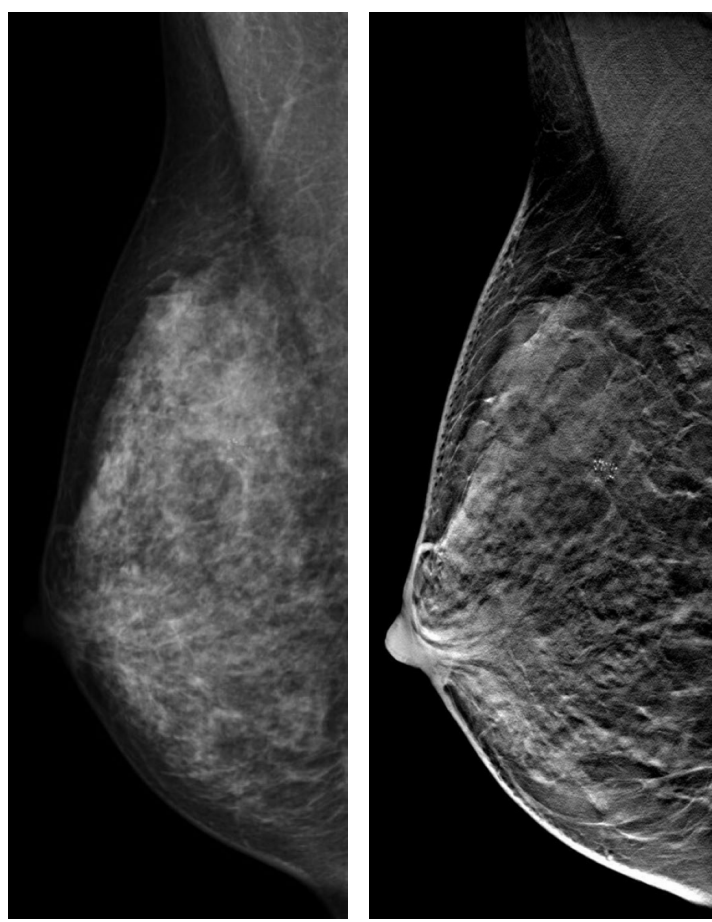

Figure 2. Images from a 53-year-old woman with ductal carcinoma in situ show amorphous to coarse heterogeneous calcifications in a grouped distribution in the right breast. (Left) Full-field digital mammography (FFDM). (Rjght) The digital breast tomosynthesis (DBT) study shows the calcification more clearly as seen on this single slice. 
the diagnosis of breast lesions. Some studies demonstrated that FFDM appears to be more sensitive than DBT for the detection of calcification. Spangler et al. reported that FFDM sensitivity was higher than DBT sensitivity (84\% and 75\%) [16]. The result is similar to that of a comparative study by Poplack et al. [18]. They showed that in $73 \%$ of the cases, microcalcifications were seen with inferior image quality with DBT. Tagliafico et al. also reported that malignant clusters of microcalcifications could be undetected with DBT but could be easily detected with FFDM [19]. They performed the study using DBT with a narrow scan-angle ( $15^{\circ}$ projections). Conversely, Kopans et al. reported that the clarity of DBT images in $92 \%$ cases was equal to or better than that of FFDM and was judged to be better than FFDM in almost half of cases [15]. A more recent study by Byun et al. compared DBT and FFDM with a wide scan-angle obtained from specimens. They also found that DBT image quality appeared to be comparable with or better than FFDM image quality in demonstrating microcalcifications [22]. These studies showed that, with an adequate image acquisition and reconstruction protocol, DBT and FFDM performance in the visualization of microcalcifications was comparable [23] [24].

Our results from using DBT with a wide scan-angle $\left(50^{\circ}\right)$ confirm the usefulness of DBT in the detection and classification of microcalcifications. A wide scan-angle allows the acquisition of more data and an increased contrast and better separation of soft tissue lesions from the normal parenchyma [21]. In the preliminary phantom study comparing narrow-angle and wide-angle DBT, narrow-angle DBT was reported to visualize a finer diameter than wide-angle DBT [25]. Our results, as well as those of other studies, support the use of DBT in the diagnoses of microcalcifications, and might suggest the possibility of using DBT as a stand-alone technique [26] [27] [28].

The visibility rating of DBT was significantly superior to that of FFDM. In addition, the visibility scores were lower for benign microcalcifications. We hypothesized this could be related to the microcalcification distribution (i.e., diffuse and regional microcalcifications may be more difficult to assess on DBT slices than segmental or grouped microcalcifications) or on the variable nature of the microcalcifications and their association with surrounding soft tissue findings, such as masses, asymmetries, or architectural distortions [29]. Microcalcifications partially obscured by normal breast tissue were not clearly visible on FFDM. DBT generates multiple projections by rotating the X-ray arm over a limited angular range. Therefore, by reducing the structural noise of the normal breast, microcalcifications that were hidden on FFDM were more clearly visible on DBT [30] [31].

Our study has a number of limitations. First, the study was a retrospective analysis of cases performed at a single center, and the patients were not randomized, which may not accurately represent the clinical problem. Second, our institution is a referral hospital which in itself might affect the study population as we have a higher proportion of patients with malignant lesions. Therefore, the 
proportion of malignancy in our cohort is higher than that in the general population. This question would require a much larger and more complex review. Third, we only tested one DBT system; consequently, our results may not be transferable to other DBT systems with detector characteristics, angular scan range, reconstruction algorithms, and image noise.

\section{Conclusion}

DBT with a wide scan-angle enables the visualization and characterization of microcalcifications at a level comparable to that of FFDM. The image quality of DBT with a wide scan-angle is comparable to or better than that obtained with FFDM, both in terms of observation and visibility of microcalcifications. Therefore, DBT can be considered appropriate for the evaluation of microcalcifications.

\section{Acknowledgements}

This work was supported by Japan Society for the Promotion of Science; Grant-in-Aid for Scientific Research (JP17K10375). We would like to thank Editage (https://www.editage.jp/) for English language editing.

\section{Conflicts of Interest}

The authors declare no conflicts of interest regarding the publication of this paper.

\section{References}

[1] Ciatto, S., Houssami, N., Bernardi, D., Caumo, F., Pellegrini, M., Brunelli, S., et al. (2013) Integration of 3D Digital Mammography with Tomosynthesis for Population Breast-Cancer Screening (STORM): A Prospective Comparison Study. The Lancet Oncology, 14, 583-589. https://doi.org/10.1016/S1470-2045(13)70134-7

[2] Skaane, P., Bandos, A.I., Gullien, R., Eben, E.B., Ekseth, U., Haakenaasen, U., et al. (2013) Comparison of Digital Mammography Alone and Digital Mammography plus Tomosynthesis in a Population-Based Screening Program. Radiology, 267, 47-56. https://doi.org/10.1148/radiol.12121373

[3] Friedewald, S.M., Rafferty, E.A., Rose, S.L., Durand, M.A., Plecha, D.M., Greenberg, J.S., et al. (2014) Breast Cancer Screening Using Tomosynthesis in Combination with Digital Mammography. JAMA, 311, 2499-2507.

https://doi.org/10.1001/jama.2014.6095

[4] Zuley, M.L., Bandos, A.I., Ganott, M.A., Sumkin, J.H., Kelly, A.E., Catullo, V.J., et al. (2013) Digital Breast Tomosynthesis versus Supplemental Diagnostic Mammographic Views for Evaluation of Noncalcified Breast Lesions. Radiology, 266, 89-95. https://doi.org/10.1148/radiol.12120552

[5] Bian, T., Lin, Q., Cui, C., Li, L., Qi, C., Fei, J. and Su, X. (2016) Digital Breast Tomosynthesis: A New Diagnostic Method for Mass-Like Lesions in Dense Breasts. The Breast Journal, 22, 535-540. https://doi.org/10.1111/tbj.12622

[6] Ohashi, R., Nagao, M., Nakamura, I., Okamoto, T. and Sakai, S. (2018) Improvement in Diagnostic Performance of Breast Cancer: Comparison between Conventional Digital Mammography Alone and Conventional Mammography plus Digital Breast Tomosynthesis. Breast Cancer, 25, 590-596. 
https://doi.org/10.1007/s12282-018-0859-3

[7] Choi, W.J., Kim, H.H., Lee, S.Y., Chae, E.Y., Shin, H.J., Cha, J.H., et al. (2016) A Comparison between Digital Breast Tomosynthesis and Full-Field Digital Mammography for the Detection of Breast Cancers. Breast Cancer, 23, 886-892. https://doi.org/10.1007/s12282-015-0656-1

[8] Seo, M., Chang, J.M., Kim, S.A., Kim, W.H., Lim, J.H., Lee, S.H., et al. (2016) Addition of Digital Breast Tomosynthesis to Full-Field Digital Mammography in the Diagnostic Setting: Additional Value and Cancer Detectability. Journal of Breast Cancer, 19, 438-446. https://doi.org/10.4048/jbc.2016.19.4.438

[9] Gur, D., Abrams, G.S., Chough, D.M., Ganott, M.A., Hakim, C.M., Perrin, R.L., et al. (2009) Digital Breast Tomosynthesis: Observer Performance Study. American Journal of Roentgenology, 193, 586-591. https://doi.org/10.2214/AJR.08.2031

[10] Good, W.F., Abrams, G.S., Catullo, V.J., Chough, D.M., Ganott, M.A., Hakim, C.M., et al. (2008) Digital Breast Tomosynthesis: A Pilot Observer Study. American Journal of Roentgenology, 190, 865-869. https://doi.org/10.2214/AJR.07.2841

[11] Gennaro, G., Toledano, A., di Maggio, C., Baldan, E., Bezzon, E., La Grassa, M., et al. (2010) Digital Breast Tomosynthesis versus Digital Mammography: A Clinical Performance Study. European Radiology, 20, 1545-1553.

https://doi.org/10.1007/s00330-009-1699-5

[12] Burnside, E.S., Ochsner, J.E., Fowler, K.J., Fine, J.P., Salkowski, L.R., Rubin, D.L., et al. (2007) Use of Microcalcification Descriptors in BI-RADS 4th Edition to Stratify Risk of Malignancy. Radiology, 242, 388-395.

https://doi.org/10.1148/radiol.2422052130

[13] Castronovo, V. and Bellahcene, A. (1998) Evidence That Breast Cancer Associated Microcalcifications Are Mineralized Malignant Cells. International Journal of Oncology, 12, 305-308. https://doi.org/10.3892/ijo.12.2.305

[14] Tabár, L., Chen, H.H., Duffy, S.W., Yen, M.F., Chiang, C.F., Dean, P.B., et al. (2000) A Novel Method for Prediction of Long-Term Outcome of Women with T1a, T1b, and 10-14 mm Invasive Breast Cancers: A Prospective Study. The Lancet, 355, 429-433. https://doi.org/10.1016/S0140-6736(00)82008-5

[15] Kopans, D., Gavenonis, S., Halpern, E. and Moore, R. (2011) Calcifications in the Breast and Digital Breast Tomosynthesis. The Breast Journal, 17, 638-644. https://doi.org/10.1111/j.1524-4741.2011.01152.x

[16] Spangler, M.L., Zuley, M.L., Sumkin, J.H., Abrams, G., Ganott, M.A., Hakim, C., et al. (2011) Detection and Classification of Calcifications on Digital Breast Tomosynthesis and 2D Digital Mammography: A Comparison. American Journal of Roentgenology, 196, 320-324. https://doi.org/10.2214/AJR.10.4656

[17] Wallis, M.G., Moa, E., Zanca, F., Leifland, K. and Danielsson, M. (2012) Two-View and Single-View Tomosynthesis versus Full-Field Digital Mammography: HighResolution X-Ray Imaging Observer Study. Radiology, 262, 788-796. https://doi.org/10.1148/radiol.11103514

[18] Poplack, S.P., Tosteson, T.D., Kogel, C.A. and Nagy, H.M. (2007) Digital Breast Tomosynthesis: Initial Experience in 98 Women with Abnormal Digital Screening Mammography. American Journal of Roentgenology, 189, 616-623.

https://doi.org/10.2214/AJR.07.2231

[19] Tagliafico, A., Mariscotti, G., Durando, M., Stevanin, C., Tagliafico, G., Martino, L., et al. (2015) Characterisation of Microcalcification Clusters on 2D Digital Mammography (FFDM) and Digital Breast Tomosynthesis (DBT): Does DBT Underestimate Microcalcification Clusters? Results of a Multicentre Study. European Radiol- 
ogy, 25, 9-14. https://doi.org/10.1007/s00330-014-3402-8

[20] Sechopoulos, I. (2013) A Review of Breast Tomosynthesis. Part I. The Image Acquisition Process. Medical Physics, 40, Article ID: 014301. https://doi.org/10.1118/1.4770279

[21] Sechopoulos, I. and Ghetti, C. (2009) Optimization of the Acquisition Geometry in Digital Tomosynthesis of the Breast. Medical Physics, 36, 1199-1207. https://doi.org/10.1118/1.3090889

[22] Byun, J., Lee, J.E., Cha, E.S., Chung, J. and Kim, J.H. (2017) Visualization of Breast Microcalcifications on Digital Breast Tomosynthesis and 2-Dimensional Digital Mammography Using Specimens. Breast Cancer (Auck), 11, 1178223417703388. https://doi.org/10.1177/1178223417703388

[23] Timberg, P., Baath, M., Andersson, I., Mattsson, S., Tingberg, A. and Ruschin, M. (2012) Visibility of Microcalcification Clusters and Masses in Breast Tomosynthesis Image Volumes and Digital Mammography: A 4AFC Human Observer Study. Medical Physics, 39, 2431-2437. https://doi.org/10.1118/1.3694105

[24] Cockmartin, L., Marshall, N.W., Van Ongeval, C., Aerts, G., Stalmans, D., Zanca, F., et al. (2015) Comparison of Digital Breast Tomosynthesis and 2D Digital Mammography Using a Hybrid Performance Test. Physics in Medicine \& Biology, 60, 3939-3958. https://doi.org/10.1088/0031-9155/60/10/3939

[25] Hadjipanteli, A., Elangovan, P., Mackenzie, A., Looney, P.T., Wells, K., Dance, D.R. and Young, K.C. (2016) Detection of Microcalcification Clusters by 2D-Mammography and Narrow and Wide Angle Digital Breast Tomosynthesis. Proceedings Medical Imaging 2016: Physics of Medical Imaging, Volume 9783, 978306. https://doi.org/10.1117/12.2217663

[26] Lång, K., Andersson, I., Rosso, A., Tingberg, A., Timberg, P. and Zackrisson, S. (2016) Performance of One-View Breast Tomosynthesis as a Stand-Alone Breast Cancer Screening Modality: Results from the Malmö Breast Tomosynthesis Screening Trial, a Population-Based Study. European Radiology, 26, 184-190. https://doi.org/10.1007/s00330-015-3803-3

[27] Durand, M.A., Haas, B.M., Yao, X., Geisel, J.L., Raghu, M., Hooley, R.J., et al. (2015) Early Clinical Experience with Digital Breast Tomosynthesis for Screening Mammography. Radiology, 274, 85-92. https://doi.org/10.1148/radiol.14131319

[28] Gilbert, F.J., Tucker, L., Gillan, M.G., Willsher, P., Cooke, J., Duncan, K.A., et al. (2015) The Tommy Trial: A Comparison of Tomosynthesis with Digital Mammography in the UK NHS Breast Screening Programme-A Multicentre Retrospective Reading Study Comparing the Diagnostic Performance of Digital Breast Tomosynthesis and Digital Mammography with Digital Mammography Alone. Health Technology Assessment, 19, 1-136. https://doi.org/10.3310/hta19040

[29] Tse, G.M., Tan, P.H., Cheung, H.S., Chu, W.C. and Lam, W.W. (2008) Intermediate to Highly Suspicious Calcification in Breast Lesions: A Radio-Pathologic Correlation. Breast Cancer Research and Treatment, 110, 1-7. https://doi.org/10.1007/s10549-007-9695-4

[30] Wheeler, F.W., Perera, A.A., Claus, B.E., Muller, S.L., Peters, G. and Kaufhold, J.P. (2006) Microcalcification Detection in Digital Tomosynthesis Mammography. SPIE Medical Imaging, San Diego, 16 February 2006, Article ID: 614420. https://doi.org/10.1117/12.653478

[31] van Schie, G. and Karssemeijer, N. (2009) Noise Model for Microcalcification Detection in Reconstructed Tomosynthesis Slices. SPIE Medical Imaging, Lake Buena Vista, 10 February 2009, Article ID: 72600M. https://doi.org/10.1117/12.810936 